\title{
INVESTIGACIONES
}

\section{Percepciones de los docentes en formación sobre la investigación educativa}

\author{
Perceptions of teachers in training regarding educational research
}

\author{
Haylen Perines, ${ }^{a}$ Javier Murillo ${ }^{b}$ \\ aDoctoranda en Educación Universidad Autónoma de Madrid \\ Telf.: (34) 914972117. Correo electrónico: profesorahaylen@gmail.com
}

${ }^{\text {b}}$ Facultad de Formación de Profesorado y Educación, Departamento de Didáctica y Teoría de la Educación,

Universidad Autónoma de Madrid

Telf.: (34) 914972833. Correo electrónico: javier.murillo@uam.es

\section{RESUMEN}

Este artículo presenta una investigación que busca conocer las percepciones que los profesores de Educación Primaria en formación tienen de la investigación educativa. Para lograrlo, se realizó un estudio cualitativo de enfoque fenomenográfico con veinte estudiantes de Magisterio en Educación Primaria de la Universidad Autónoma de Madrid. Los resultados, en contraste con estudios anteriores, apuntan a que los futuros profesores perciben a la investigación como un saber importante y necesario para su profesión y valoran positivamente el trabajo de los investigadores. Sin embargo, creen que su formación en investigación debe ser mejor y más útil para su desempeño docente.
\end{abstract}

Palabras clave: docentes, investigación, difusión de conocimientos, formación de profesores.

\begin{abstract}
This article presents a research that seeks to identify the perception of in training-Primary Education teachers regarding the educational research. To develop this research, a qualitative study with a phenomenographic approach was applied to twenty students of the Elementary Education Program from Universidad Autónoma de Madrid. The results, in contrast to previous studies, suggest that future teachers perceive research as important and necessary in their profession and value positively the work of researchers. However, they believe that the training they receive in terms of research should be better and more useful for their teaching performance.
\end{abstract}

Key words: teachers, research, dissemination of knowledge, teacher education. 


\section{INTRODUCCIÓN}

En el año 1993, el profesor Carl Kaestle, por entonces presidente de la Asociación Americana de Investigación Educativa (AERA), publicó un artículo que hizo temblar los cimientos de la investigación educativa. El título del mismo ya era un presagio de su contenido "The awful reputation of education research" (la terrible reputación de la investigación educativa). Su argumento era sencillo, la investigación no estaba consiguiendo su objetivo - contribuir a mejorar la educación—, y tenía un pésimo prestigio entre docentes, directivos y administradores.

Pocos años después, el influyente profesor e inspector educativo británico David Hargreaves (1996), en la conferencia anual en la Agencia de Formación de Profesorado (TTA), profundizó en ese mismo planteamiento. Así, afirmó que mientras los médicos, los ingenieros y arquitectos, incluso los agricultores en países desarrollados, toman sus decisiones apoyándose en las últimas evidencias empíricas disponibles, los profesionales de la educación lo hacen a partir de su experiencia, su sentido común, las modas, pero no de lo que la investigación ha ido legando.

Estas dos aportaciones abrieron un fuerte debate a los dos lados del Atlántico. Así, en los años 1997 y 1998 se publicaron en la revista Educational Researcher una serie de artículos por diferentes expresidentes de la AERA como respuesta a la provocación de Kaestle (p.ej., Glass, 1998; Howe, 1998; Kennedy, 1997; Labaree, 1998; Soufre, 1997; Wagner, 1997), a los que siguió una gran cantidad de trabajos que llegan hasta nuestros días; tanto en Estados Unidos (p.ej. Berliner, 2002; Burkhardt \& Schoenfeld, 2003; Feuer, Towne \& Shavelson, 2002; Hammersley, 2002, 2014; Slavin, 2002), como en Reino Unido (Edwards, 2000; Thomas \& Pring, 2004; Tooley \& Darby, 1998; Trinder \& Reynolds, 2000), y otros países, incluido España (De la Orden \& Mafokozi, 1999; Muñoz-Repiso, 2004, 2005; Murillo, 2006, 2011; Sancho, 2010).

Con todo ello se dio un importante impulso a una línea de investigación que busca profundizar en la relación entre investigación y práctica educativa y aportar ideas para su mejora (Anwaruddin, 2015; Beycioglu, Ozer \& Ugurlu, 2010; Broekkamp \& van HoutWolters, 2007; Drill, Miller \& Behrstock-Sherratt, 2012; Ion \& Iucu, 2014; Gore \& Gitlin, 2004; Hemsley-Brown \& Sharp, 2003; Levin 2013; Lysenko et al., 2014; Montgomery \& Smith, 2015; Vanderlinde \& van Braak, 2010; Winch, Oancea \& Orchard, 2015; Witherow, 2011; Zeuli, 1994). Dada la importancia de la formación del profesorado en la comprensión y mejora de esa relación, algunos trabajos se han centrado específicamente en la relación que los futuros docentes tienen con la investigación educativa (Ball \& Forzani, 2009; Counsell et al., 2000; Elliot, 2001; Korthagen, 2007; Rust, 2009; Tom, 1997; Zeichner, 1995, 2010).

En este artículo se presenta una investigación que busca conocer las visiones que los docentes de Educación Primaria en formación tienen de la investigación educativa.

\section{MARCO TEÓRICO}

Los resultados de los estudios empíricos que buscan conocer las opiniones de los docentes en formación sobre la investigación educativa (p. ej., Demircioglu, 2008; Gitlin et al., 1999; Gray, 2013; Joram, 2007; Jyrhämä et al., 2008; MacDonald, Badger \& Whites, 2001; 
Pendry \& Husbands, 2000; van der Linden et al., 2012, 2015) han destacado tres grandes temas: la imagen de la investigación como un conocimiento alejado de la realidad, la percepción crítica de los programas de formación sobre estas temáticas y la necesidad de que la investigación proporcione recursos prácticos vinculados con la experiencia.

Un primer conjunto de trabajos ha encontrado que los docentes en formación tienen una imagen un tanto negativa acerca de la investigación educativa (Gitlin et al., 1999; MacDonald et al., 2001). Así, una opinión generalizada entre los estudiantes de programas de formación docente es que la investigación es un conocimiento abstracto, alejado de la realidad y difícil de utilizar en la práctica. La opinión sobre los investigadores tampoco es muy alentadora, debido a que son observados como profesionales que realizan un trabajo alejado de la práctica. Esto genera una sensación de desconfianza hacia sus afirmaciones. Específicamente, en el estudio de Gitlin et al. (1999), la mayor parte de los futuros profesores no cambian sus puntos de vista sobre la naturaleza de la investigación al final del programa en el que participaron, ya que la siguen considerando como un conocimiento teórico que poco tiene que ver con las exigencias de la práctica.

Pero no todos los estudios dan esa imagen tan negativa de los futuros docentes hacia la investigación. Pendry y Husbands (2000), en un trabajo que se proponía determinar si la lectura de artículos de investigación por parte de 141 futuros docentes puede ser un aporte para el conocimiento que tienen de la investigación, encontró que los profesores en formación tienen una visión más bien positiva de la investigación, indicando que es un aprendizaje necesario para su preparación como profesionales porque les permite ampliar y actualizar sus conocimientos sobre la educación. Sin embargo, la lectura de artículos sigue siendo muy limitada entre ellos porque no tienen posibilidades concretas de estudiarlos en sus asignaturas, lo que es un obstáculo para que la consulten de manera habitual.

El análisis crítico de los programas de formación de profesorado es el segundo gran tema identificado en la revisión de la literatura. Los docentes en formación piensan que en su formación inicial conocen algunas investigaciones de manera superficial (Gitlin et al., 1999) lo que les parece contradictorio con su óptima preparación. Los estudiantes esperan desarrollar un mayor contacto con la investigación durante la carrera y les gustaría tener más oportunidades de participar en ella de manera activa. El estudio de Ismail Hakki Demircioglu (2008), que examina las actitudes que 74 estudiantes de pedagogía tienen hacia un curso de métodos de investigación, menciona la importancia respecto de la revisión y modificación de los programas de grado, buscando dar una mayor presencia y relevancia a las asignaturas que trabajan las etapas y fundamentos de la investigación. De acuerdo a MacDonald et al. (2001), reconciliar los discursos divergentes de la investigación y de la práctica educativa requiere de una formación de calidad en temas y metodologías investigativas.

También surgen algunas valoraciones más optimistas sobre los cursos de investigación donde han participado los futuros docentes. Por ejemplo, en el estudio de Demircioglu (2008), los participantes destacan que, a través del curso realizado, aprenden habilidades básicas de investigación educativa, como identificar problemas de investigación, realizar una revisión bibliográfica, seleccionar una metodología y elegir los instrumentos de recolección de datos, lo que les permite valorar en mayor medida a la investigación educativa y tener una mejor imagen de ella. Particularmente, los docentes en formación del estudio de Jyrhämä et al. (2008) evalúan positivamente el enfoque basado en la investigación que han conocido en el grado, y lo señalan como un tema prioritario en la organización de su formación docente. 
Por último, algunos trabajos (Gitlin et al., 1999; MacDonald et al., 2001) han destacado la opinión de los docentes en formación acerca de la necesidad de que la investigación proporcione recursos prácticos para la mejora de la docencia. El estudio desarrollado por Andrew Gitlin et al. (1999), por ejemplo, analiza la forma en que la investigación educativa impacta en el trabajo de 37 docentes en formación al principio y al final de un programa de investigación. MacDonald et al. (2001), por su parte, analizan la influencia que un curso de investigación educativa tiene en las visiones que 55 profesores en formación. Para los participantes de los dos estudios mencionados, los objetivos de la investigación son prácticos en la medida en que identifican sugerencias concretas que refuercen y mejoren inmediatamente la práctica docente. Los docentes asumen que pueden desarrollar un compromiso con la investigación siempre y cuando les dé la posibilidad de aplicar sus resultados a los contextos donde se desempeñarán en el futuro. Para Gitlin et al. (1999), la preferencia por la información práctica puede estar relacionada con la estructura convencional del plan de estudios de formación del profesorado, la que en ocasiones otorga mucha importancia al aspecto teórico de los aprendizajes por sobre sus aplicaciones prácticas. Como respuesta, los futuros docentes buscan elementos más pragmáticos y posibles de utilizar concretamente (MacDonald et al., 2001).

\section{METODOLOGÍA}

El objetivo de este estudio es conocer las percepciones que los docentes en formación de Educación Primaria tienen de la investigación educativa.

Para ello se utilizó la Fenomenografía como enfoque metodológico (Bowden \& Walsh, 2000; Marton, 1986; Richardson, 1999). Con carácter general, esta aproximación metodológica busca "identificar de forma cualitativa las diferentes formas en que las personas experimentan, conceptualizan, perciben y comprenden varios aspectos del fenómeno y de su mundo próximo" (Marton, 1986, p. 31). Así, desde esta perspectiva, el interés del investigador no se centra en los fenómenos en sí mismos, sino en cómo los sujetos vivencian y perciben dichos fenómenos.

Las categorías de análisis a priori definidas son las visiones que los futuros maestros tienen de la investigación educativa, la importancia que dan a la investigación para su futuro desempeño como profesores, las opiniones que tienen sobre la formación en investigación que han recibido y las opiniones que tienen de los investigadores educativos.

Los participantes son 20 estudiantes del grado de Magisterio en Educación Primaria de la Universidad Autónoma de Madrid. El criterio de selección de los participantes fue el año que cursaban, entrevistando a cinco estudiantes de cada nivel desde primero a cuarto año. Dentro de los cinco estudiantes de tercero, tres de ellos ya habían cursado la asignatura de metodología de la investigación (Tabla 1). 
Tabla 1. Participantes del estudio

\begin{tabular}{|l|l|}
\hline \multicolumn{1}{|c|}{ Curso } & \multicolumn{1}{|c|}{ Participantes } \\
\hline $\begin{array}{l}\text { Primer curso del grado de Magisterio } \\
\text { en Educación Primaria }\end{array}$ & 5 estudiantes \\
\hline $\begin{array}{l}\text { Segundo curso del grado de Magisterio } \\
\text { en Educación Primaria }\end{array}$ & 5 estudiantes \\
\hline $\begin{array}{l}\text { Tercero curso del grado de Magisterio } \\
\text { en Educación Primaria }\end{array}$ & $\begin{array}{l}3 \text { estudiantes que ha cursado la asignatura de } \\
\text { Metodología de Investigación }\end{array}$ \\
\cline { 2 - 2 } & $\begin{array}{l}\text { 2 estudiantes que no ha cursado la asignatura } \\
\text { de Metodología de Investigación }\end{array}$ \\
\hline $\begin{array}{l}\text { Cuarto curso del grado de Magisterio } \\
\text { en Educación Primaria }\end{array}$ & 5 estudiantes \\
\hline
\end{tabular}

Fuente: elaboración propia.

El instrumento que utilizamos es la entrevista fenomenográfica. En esta se plantearon a los estudiantes interrogantes como las siguientes: ¿qué piensas de la investigación educativa?, ¿qué piensas de su utilidad para tu futuro trabajo, ¿cómo percibes el trabajo que realizan los investigadores? El trabajo de campo se desarrolló a lo largo del curso 2014-2015.

El análisis de los datos se fundamentó en el establecimiento de ciertas categorías previas que se fueron complementando con aquellos nuevos elementos que surgían desde los propios datos recabados. En el transcurso de la investigación nos apoyamos en el programa Atlas.ti, que nos facilitó la codificación y jerarquización de los datos empíricos. Los códigos ya jerarquizados y agrupados en familias se indican en la matriz hermenéutica (Tabla 2).

Tabla 2. Matriz hermenéutica de las visiones de la investigación para docentes en formación

\begin{tabular}{|l|l|}
\hline Familias semánticas & \multicolumn{1}{|c|}{ Códigos asociados en orden de frecuencia } \\
\hline $\begin{array}{l}\text { Concepto de } \\
\text { investigación } \\
\text { educativa }\end{array}$ & $\begin{array}{l}\text { Herramienta fundamental y necesaria/Asociación entre investigación y } \\
\text { continuación de estudios de posgrado/Proceso científico basado en } \\
\text { hipótesis/Método riguroso y planificado/Conocimiento en profundidad de } \\
\text { algún fenómeno }\end{array}$ \\
\hline $\begin{array}{l}\text { Utilidad de la } \\
\text { investigación } \\
\text { educativa }\end{array}$ & $\begin{array}{l}\text { Herramienta que identifica problemas/Solución de problemas /Adquisición } \\
\text { de conocimientos actualizados/Conocimiento de metodologías innovadoras/ } \\
\text { Profesionalización de la docencia/Visión optimista del aporte de la } \\
\text { investigación a la docencia/Mirada objetiva de los problemas/Diagnosticar } \\
\text { problemas/Evaluar procesos/Actualización de los conocimientos/Confirmar } \\
\text { o rechazar creencias/Ayudar al progreso de la educación }\end{array}$ \\
\hline
\end{tabular}




\begin{tabular}{|l|l|}
\hline $\begin{array}{l}\text { Perspectivas del rol } \\
\text { del investigador }\end{array}$ & $\begin{array}{l}\text { Investigador como persona importante/Valoración positiva del trabajo del } \\
\text { investigador/Persona importante para el progreso de la educación/ }\end{array}$ \\
\hline Actitud del profesor & $\begin{array}{l}\text { Poca disposición del docente frente a la lectura de estudios/Situación } \\
\text { generacional de los profesores }\end{array}$ \\
\hline $\begin{array}{l}\text { Visión de la } \\
\text { formación de grado } \\
\text { recibida }\end{array}$ & $\begin{array}{l}\text { Visión crítica de su formación en investigación/Necesidad de recibir } \\
\text { formación en investigación/ Preocupación por la escasez de lecturas } \\
\text { científicas/Líderes de las escuelas como posibles difusores de los estudios/ } \\
\text { Conocer la investigación a través del trabajo en equipo/ }\end{array}$ \\
\hline
\end{tabular}

Fuente: elaboración propia.

\section{RESULTADOS}

En esencia, es posible distinguir cinco grandes temas en el discurso de los participantes (Figura 1): concepto de investigación educativa, utilidad de la investigación educativa, actitud del docente hacia la investigación educativa, visión del investigador y visión de la formación en investigación. Organizaremos la presentación de los resultados agrupándolos en estos grandes bloques.

Figura 1. Categorías descriptivas de las visiones de los docentes en formación

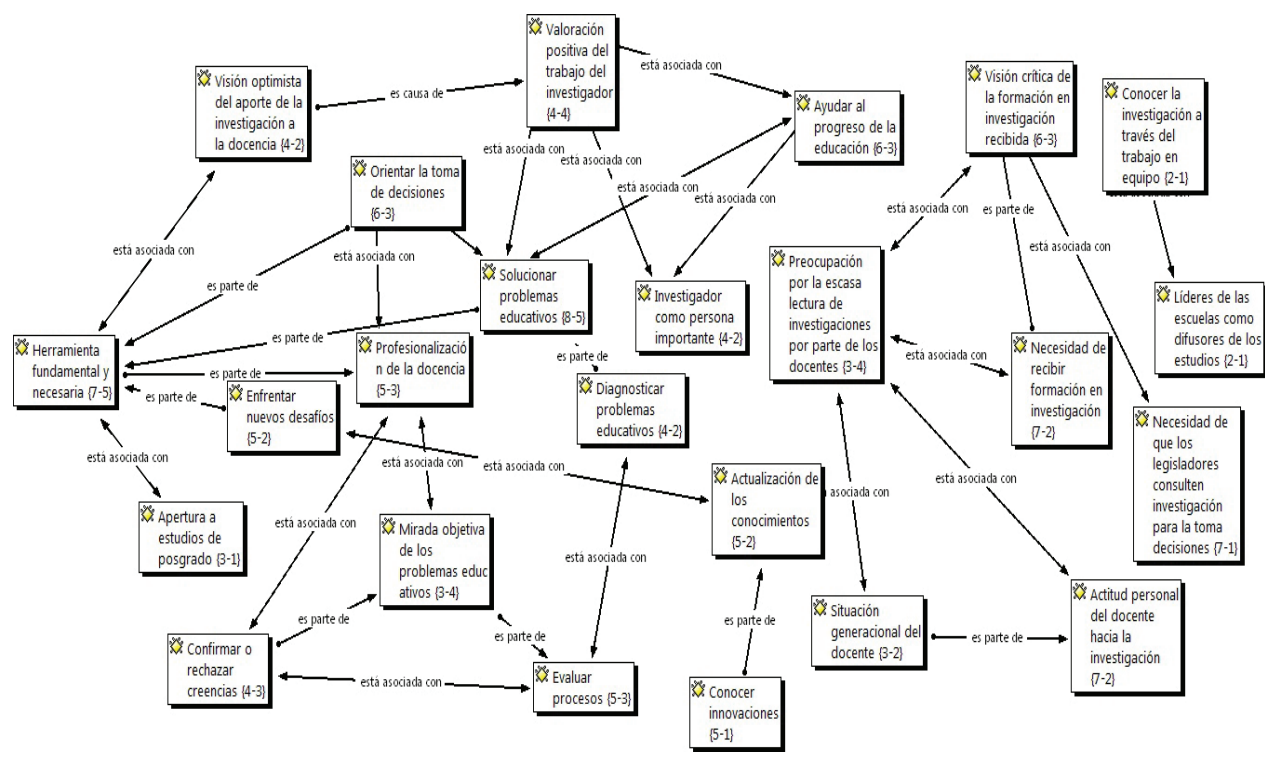

Fuente: elaboración propia. 


\subsection{EL CONCEPTO DE INVESTIGACIÓN EDUCATIVA}

Como parece razonable, el concepto que tienen los estudiantes del grado de Maestro en Educación Primaria acerca de qué es investigación educativa en diferente en función del curso que realizan. Los del primer y segundo año tienen definiciones de investigación más superficiales y generales que los de tercero y cuarto. No conocen en profundidad el concepto pero se esfuerzan en intentar esbozar algunas ideas:

La investigación puede servir para que conozcamos nuevas formas de hacer las cosas, quizás cosas más creativas, más novedosas, menos rutinarias y menos monótonas de hacer la cosas. Yo lo miro así... la educación día a día enfrenta problemas y hay que buscar formas de solucionarlos, la investigación puede tener gran utilidad para eso. (DF3, S: 8) ${ }^{1}$.

Una de las estudiantes de segundo año realiza una asociación entre la adquisición de conocimientos sobre la investigación educativa y la posibilidad de continuar estudios de posgrado. Para ella, quienes se dedican a profundizar aspectos de la investigación son aquellas personas que cursan o pretenden cursar estudios de máster o doctorado. La estudiante valora positivamente la continuación de los estudios y el seguir profundizando en temas educativos a través de la investigación:

Me parece bien que haya gente que quiera seguir estudiando y avanzando en sus carreras. Ir creciendo profesionalmente debería ser una aspiración de todos quienes se dedican a la educación. Mientras más preparados estamos, es mejor para nuestros estudiantes. (DF5, S: 16).

Por su parte, los estudiantes de tercer año esbozan una definición más profunda y detallada de la investigación educativa, en la que incluyen conceptos más específicos de la misma. Estos participantes asocian la investigación con un proceso científico basado en hipótesis que buscan ser verificadas o rechazadas, lo que requiere de la observación constante de la realidad.

Los futuros profesores de tercer año que ya han cursado la asignatura de metodología de investigación amplían un poco más el concepto. Ellos reiteran algunos de los términos mencionados por los estudiantes de primero y segundo, pero agregan que la investigación es un sistema organizado de búsqueda de información basada en un método riguroso y planificado llevado a cabo por personas especializadas. El método sigue ciertas etapas organizadas previamente, las que deben ser ejecutadas con profesionalismo y seriedad:

La investigación se basa en criterios de orden científico. Se plantea una idea que se busca indagar, comprobar, ver si es verdadera y para eso los investigadores se acercan a la realidad y prueban las hipótesis. No es un proceso desordenado sino que sigue ciertas etapas ordenadas de acuerdo a una línea. (DF11, T: 39).

De este modo se señala la línea de la entrevista, derivada de la transcripción y análisis en el software Atlas.ti. Las letras DF son la abreviación de la expresión docente en formación. Las letras P, S, T y C son la abreviación de primero, segundo, tercero o cuarto año de formación respectivamente. 
Los estudiantes de cuarto año tienen conocimientos más amplios y detallados de la investigación educativa en comparación con los estudiantes de primero, segundo o tercero. Esta situación puede ser explicada no solo por su formación en metodología de investigación, sino también porque han hecho prácticas en centros educativos, tienen un mayor bagaje de conocimientos acerca de su futura profesión producto del avance paulatino en la carrera y se están enfrentando a la realización del Trabajo Fin de Grado:

La investigación educativa se basa en criterios de orden científico y en ella son relevantes conceptos como el tipo de estudio, el diseño, la muestra, etc.; al igual que la recopilación de antecedentes para establecer hipótesis acerca del tema. Parte de la idea de que hay que observar la realidad para detectar un tema que sea interesante, que plantee dudas. (DF16, C: 56).

\subsection{PERCEPCIONES ACERCA DE LA UTILIDAD DE LA INVESTIGACIÓN EDUCATIVA}

Las percepciones que los futuros profesores tienen de la utilidad de la investigación educativa son muy parecidas, con independencia del nivel que cursan. Entre ellos predomina la idea de que la investigación es un recurso de gran utilidad y la describen como un material fundamental, necesario y esencial en los procesos educativos. Observan a la investigación como una herramienta que permite identificar situaciones que necesitan ser optimizadas:

La investigación no solo debe servir para buscar métodos nuevos sino que también para evaluar lo que ya se ha hecho y ver si está bien o está mal. De esa manera es posible analizar de manera crítica las situaciones educativas, pero de una manera crítica que sea constructiva, positiva y con el objetivo de mejorar algún aspecto deficiente. (DF15, T: 53).

Al mismo tiempo, los estudiantes piensan que, al diagnosticar dificultades del sistema, la investigación facilita un cambio educativo que supere modelos utilizados tradicionalmente en la escuela. Incluso, la utilización responsable de la investigación puede llevar a proponer cambios que impliquen transformaciones en los paradigmas establecidos, destacando la importancia de que haya modificaciones sustanciales en los esquemas educativos existentes. Desde la perspectiva de estos participantes es importante observar críticamente las prácticas educativas. Con el apoyo de la investigación se pueden generar instancias de autocrítica dentro de los centros escolares donde las instituciones observen su realidad, su organización, sus formas de funcionamiento y su estructura desde la perspectiva de los investigadores educativos:

La investigación debe ayudar a que los centros miren lo que se ha descubierto o lo que se ha analizado acerca de temas que les aquejan y nutrirse de esa nueva información... y si se están haciendo mal las cosas, comenzar a cambiarlas. (DF12, T: 42).

Algunos de los participantes conciben a la investigación como un instrumento que permite a los educadores estar siempre atentos a los cambios metodológicos que surgen en la literatura y a la forma global en que se entiende el concepto de educación. Para ellos, el 
crecimiento y desarrollo profesional de los docentes debe ir de la mano con los avances en la investigación. Sus palabras son las siguientes:

La educación va cambiando y la investigación nos debería ayudar para estar al tanto de estos cambios y no quedarnos atrás. Las nuevas generaciones vemos que las cosas van cambiando con mucha prisa, entonces las investigaciones que surgen hoy en día deberían ser útiles para eso, para que los profesores estén actualizados y enterados de los avances en materias de educación. (DF15, T: 53).

Los entrevistados consideran que la investigación educativa es fundamental en el proceso de enseñanza-aprendizaje, porque permite dar una mirada más objetiva de las problemáticas educativas. Un docente puede diagnosticar problemas que se dan en su aula de manera intuitiva, pero para solucionarlos no solo requiere de su experiencia o sentido común sino que también necesita la rigurosidad de la investigación:

Un profesor puede saber y detectar que existe un problema educativo o una situación que requiere apoyo, sin embargo, es necesaria la rigurosidad científica por medio de investigaciones que den otra mirada de la misma situación. No basta nuestra intuición, la que es muy válida, pero también la investigación hace un gran trabajo que debe ser aprovechado por las personas que trabajan en la educación. (DF20, C: 71).

Para los estudiantes de Magisterio, la investigación debe ayudar a derribar o confirmar mitos instalados en las aulas y en las formas de pensar de los profesores. Algunos de ellos señalan que, en ocasiones, los docentes se apoyan en pensamientos que se van instalando fuertemente en su quehacer, pasando de generación en generación y permaneciendo en una especie de cultura colectiva difícil de cambiar. Estas creencias pueden generar una mirada muy cerrada de parte de los profesores frente a ciertos acontecimientos, ya que favorecen los estereotipos y las ideas no fundamentadas con suficientes respaldos:

Los mitos existen mucho en educación, esas ideas cerradas que se transmiten de generación en generación, que van de boca en boca y se convierten en una verdad. Quizás esas ideas deben ser más analizadas y más criticadas, para eso hay que mirar estudios que permitan abrir un poco las perspectivas. (DF17, C: 62).

Una opinión generalizada entre los entrevistados es la asociación de la investigación educativa con la posibilidad de conocer innovaciones educativas, especialmente nuevas habilidades, estrategias y metodologías. Los estudiantes esperan que la investigación les ayude a superar la estructura fija y el prototipo de educación tradicional que aún impera en las aulas, centrada en los libros de textos, en la enseñanza vertical, homogénea y poco abierta a la diversidad:

La investigación puede servir para estar al tanto de nuevas metodologías quizás más dinámicas, más ágiles, que puedan ayudar a la atención a la diversidad y a saber educar a los chicos que tiene alguna discapacidad. No todos los alumnos aprenden igual y, hasta la fecha, la educación no se hace cargo de esas situaciones. (DF15, T: 55). 


\subsection{PERCEPCIONES SOBRE LAS ACTITUDES DE LOS DOCENTES FRENTE A LA INVESTIGACIÓN EDUCATIVA}

Una parte de los participantes considera que consultar investigación depende, en gran medida, de la iniciativa personal de cada persona. Desde la perspectiva de los estudiantes de Magisterio, un profesor que quiere optimizar sus prácticas, actualizarse y adquirir nuevos conocimientos debería interesarse por leer artículos de forma periódica. Si los docentes tienen una actitud positiva hacia los estudios, pueden contar con mejores recursos para desarrollar su profesión. Dos testimonios de estudiantes de tercero y cuarto señalan lo siguiente:

Es cierto que la investigación será importante para quien quiera realmente mirarla y leerla. O sea, también depende de la iniciativa de cada profesional. Si eres autónomo y quieres irte actualizando vas a consultar estudios y cosas así que te permitan hacer mejor tu trabajo. (DF9, T: 31).

Leer artículos también depende de cada persona, si tienes un mínimo de interés y de respeto por lo que estás haciendo y de verdad valoras lo que haces, te exiges a ti mismo el aprender más, el reciclarte y el perfeccionarte. (DF19, C: 69).

Una de las estudiantes de tercero, si bien es crítica con la excesiva pasividad de algunos docentes frente a la adquisición de nuevos conocimientos, reconoce que es algo que también puede pasarle a ella y a sus compañeros cuando ingresen al mundo laboral. Para ella, en la etapa en que se encuentran actualmente están con más energía y entusiasmo frente al aprendizaje contante, pero con el tiempo y las experiencias dicha energía puede ir decayendo:

Hay profesores muy cómodos, no digo que nosotros no llegaremos nunca a ser así, quizás también nos suceda, pero al ser cómodos prefieren seguir repitiendo siempre la misma rutina sin leer nada nuevo. Simplemente, si no te interesa, no leerás nada de estudios y artículos. Pero también es cierto que nuestra posición actual nos da más energía, más vitalidad, ojalá con los años nos podamos mantener así y que nuestra buena disposición no decaiga. (DF13, T: 47).

Para otros de los entrevistados, la actitud del docente hacia la investigación también puede estar relacionada con la dinámica del centro, ya que la presencia de un grupo de docentes que se interesa por leer y consultar estudios puede influir positivamente en los demás para que también lo hagan. Lo mismo sucede con las decisiones del equipo directivo del centro: en los claustros o reuniones de departamento pueden utilizarse activamente resultados de investigaciones o artículos recientes, lo que debe ser gestionado por el propio equipo directivo:

He visto en mis prácticas que hay centros muy involucrados y comprometidos con utilizar investigaciones para buscar nuevas estrategias educativas, también depende un poco de cómo el equipo directivo gestiona sus reuniones. Con un pequeño esfuerzo quizás el director puede mostrar a los profesores resultados de algún estudio y así la palabra investigación ya no les parece tan ajena. (DF12, T: 43).

Otra de las temáticas que surge al analizar la actitud de los profesores es la edad que tiene cada profesor y sus años de experiencia docente. De acuerdo a algunos de los estudiantes, 
la importancia que cada docente le dé a la investigación en su forma de ejercer la profesión depende de la etapa generacional en la que se encuentra. Para ellos, los profesores con más experiencias o con más edad recibieron una instrucción basada en métodos más antiguos, clásicos y tradicionales. En consecuencia, será mucho más difícil que estos profesionales lean estudios. En cambio, los docentes más nóveles y ellos mismos como profesores en formación pueden sentirse más cercanos a las novedades e innovaciones metodológicas como parte de una actitud más receptiva y abierta hacia nuevos recursos. Otro factor que influye en las diferencias generacionales es el manejo absoluto de Internet que tienen los futuros docentes y los profesores jóvenes, que es el mecanismo más idóneo para consultar investigaciones de forma fácil e inmediata:

Entonces, si me pongo en el lugar de los profesores que ejercen pues imagínate, seguro que ellos estudiaron hace mucho tiempo donde lo que más leían eran libros, ten por seguro que casi no conocieron artículos. Pero la idea es que eso cambie y los futuros profesores conozcamos mejor la investigación para que nos apoyemos en ella cuando estemos ejerciendo. (DF14, T: 52).

Por otra parte, los futuros profesores se muestran preocupados frente a la falta de apoyo de parte de las políticas públicas para disminuir la distancia entre la investigación y la práctica docente. Para algunos estudiantes las Administraciones deben velar por una mejor utilización de los resultados de la investigación en las escuelas. Estas deberían apoyar sus decisiones en los hallazgos de los estudios, teniendo como prioridad dar solución a los problemas educativos a la luz de la investigación. Las decisiones públicas en materias de educación deberían leer y escuchar en mayor medida los resultados de las investigaciones, ya que de esta forma podrían planificar mejor las leyes, reformas y medidas adoptadas:

Muchos de los fracasos en materias de educación pública son porque los gobiernos no escuchan los aportes de las investigaciones. Si los estudios fueran más escuchados las cosas podrían mejorar. (DF18, C: 65).

\subsection{PERCEPCIONES SOBRE LOS PROFESIONALES DE LA INVESTIGACIÓN}

Los docentes en formación tienen una opinión bastante positiva sobre los investigadores educativos, valorando su trabajo y lo que significan para la sociedad. Desempeñarse como investigador no les parece una tarea fácil, ya que requiere de gran experticia en el área, además de minuciosidad, rigurosidad y posesión de un afán científico que no todas las personas logran desarrollar:

Los investigadores hacen en un arduo trabajo en el cual es importante el carácter objetivo para conocer los problemas a fondo y acercarse a la verdad. La objetividad le da una mirada más seria a los problemas, y lograr ser objetivo no es una tarea fácil, entonces no cualquier persona puede ser investigador. (DF20, C: 67).

Desde la visión de los estudiantes de Magisterio, los investigadores son profesionales que aportan elementos importantes para el perfeccionamiento de las prácticas educativas. 
Destacan que detrás de una investigación existe un trabajo metódico y sistemático realizado por un profesional que busca alcanzar resultados fiables.

Aunque los futuros docentes valoran positivamente el rol de los investigadores, también mencionan algunas impresiones más críticas acerca de su trabajo. Un grupo de ellos afirma que los investigadores viven en un plano demasiado académico, elevado y poco cercano al docente de aula, lo que limita su acercamiento a la realidad de las aulas y una mayor difusión de su trabajo:

También es cierto que algunos investigadores están en otro contexto, muy diferente al de los profesores. Entonces no existe comunicación ni tampoco conexión entre ellos. El investigador está en un plano y el profesor está en otro. No interactúan, no hablan, no dialogan lo que debe cambiar. (DF19, T: 68).

Los estudiantes afirman que como profesores en formación tienen mayores posibilidades de interactuar con los investigadores en comparación a un profesor en ejercicio, ya que están inmersos en su contexto y cotidianidad. Al estar en la universidad reciben clases de parte de los investigadores, los sienten más cercanos y los perciben como personas accesibles para dialogar e intercambiar opiniones:

En la Universidad hay más posibilidades de ver a los investigadores como personas más cercanas, más normales. O sea, ellos son quienes nos dan las clases entonces podemos hacerles preguntas, tocar diversos temas, debatir y conocer los estudios que hacen. Incluso nos han hecho leer sus propias investigaciones. (DF18, T: 50).

Desde la perspectiva de estos participantes, la relación más cercana que pueden establecer los futuros docentes con los investigadores es una posibilidad que tienen de manera casi exclusiva, ya que los docentes en ejercicio no cuentan con estas oportunidades, al estar inmersos en su trabajo de aula en contextos externos a la universidad:

Si un profesor que trabaja en un colegio está por allí todo el tiempo, prácticamente no conocerá lo que hacen los investigadores. Es diferente cuando estamos acá en la uni, la relación con lo que ellos hacen es mejor y un poco más habitual. (DF10, S: 35).

\subsection{OPINIONES HACIA SU PROPIA FORMACIÓN EN INVESTIGACIÓN}

En términos generales, los estudiantes del grado de maestro que han recibido formación en métodos de investigación son muy críticos con ella. Para ellos, dicha formación debería ser sustancialmente mejor y más útil para su desempeño docente. Si bien valoran que han leído algunos estudios y que han conocido los elementos esenciales de la investigación, piensan que pueden existir iniciativas más tempranas para profundizar en esta. Es interesante que los estudiantes de tercero que aún no cursan las asignaturas de metodologías de investigación vean con preocupación que recién las vayan a tener en el siguiente curso, ya que les parece un poco tardío para lograr familiarizarse realmente con ella:

El año que viene tenemos que hacer una investigación y estamos un poco perdidos.

Nos falta más relación con la investigación. Conocemos muy poco, casi nada, solo hay 
una asignatura lo cual no es suficiente para formar adecuadamente a una persona en el tema. Si bien no todos queremos ser investigadores sí necesitamos saber más de ella para utilizarla más en nuestro futuro. (DF14, T: 51).

Los estudiantes de tercero y cuarto que ya han tenido la asignatura de metodología valoran positivamente lo que han aprendido en ella, sin embargo, creen que para aprender a utilizarla deben conocerla desde los primeros años de estudio. Estos participantes critican que en primero y segundo no hayan conocido nada de investigación, ya que esperarían tener un contacto más temprano con ella para ir acercándose a sus características y funciones. Sería ideal que desde primer año de carrera tuvieran contacto con artículos y resultados de estudios, quizás no de una manera tan sistemática como en la asignatura correspondiente, pero sí como parte de una iniciación en el tema. De esta manera podrían incorporar la investigación a sus conocimientos:

Es cierto que en tercero y cuarto tenemos mayor madurez para entender más de estudios de investigación, pero quizás desde primero puede haber mayor acercamiento, contactos breves pero importantes que logren hacer que nos vayamos interesando en el tema. (DF17, C: 61).

Una de las futuras profesoras señala que en Magisterio debería existir una cultura diferente en torno a la investigación, capaz de fomentar una profesionalización de la carrera y un mejor nivel de preparación de los estudiantes:

Si se formara mejor a los docentes, la solución a los problemas educativos sería más profesional. Es cierto que cada problema es único, pero lo que vi en mis prácticas es que a veces se usa lo que dice la mayoría y punto, sin apoyarse en lo que otras personas ya han descubierto acerca de ese problema. (DF18, C: 65).

De acuerdo a uno de los participantes, aprender más de la investigación no quiere decir que en la carrera se reemplace la formación específica de un máster. El objetivo es que se fomente un mayor uso de la investigación como recurso útil y actualizado para el actuar docente. Sus palabras son las siguientes:

No deseamos convertirnos en investigadores expertos, pero eso no quiere decir que no vamos a querer utilizar los recursos que proporciona la investigación educativa para mejorar las prácticas. (DF11, T: 40).

\section{DISCUSIÓN}

En términos generales, nuestros hallazgos sobre la percepción que tienen los futuros docentes acerca de la investigación educativa son más positivos que los encontrados en algunos trabajos anteriores (p. ej., Gitlin et al., 1999; Gray, 2013; MacDonald et al., 2001). Esta discrepancia se disipa cuando se contrasta la imagen que los estudiantes de Magisterio tienen sobre la formación que reciben en investigación o cuando se analiza la vinculación entre la investigación y la práctica, ya que en estos tópicos vemos importantes puntos de encuentro. 
Si comenzamos por las diferencias entre nuestros hallazgos y los estudios previos vemos que los profesores en formación de este estudio expresan opiniones positivas sobre la investigación, al contrario, por ejemplo, de lo hallado en el estudio de MacDonald et al. (2001) donde los docentes expresan que la investigación es un conocimiento excesivamente teórico que no logra influir en las personas que trabajan en las escuelas. Entre estas opiniones críticas surge una voz más optimista que coincide con la de este estudio, nos referimos al estudio de Pendry y Husbands (2000) donde los participantes expresan que la investigación es un recurso muy importante para lograr cambios en la educación, por lo que debe ser utilizada con mayor frecuencia por el profesorado.

Una diferencia similar se aprecia en la imagen que se tiene del investigador. Los estudiantes de Magisterio son muy generosos con sus comentarios hacia estos profesionales, en cambio, en los hallazgos de investigaciones anteriores predomina la idea de que son personas lejanas al contexto real de las escuelas (Gitlin et al., 1999).

Encontramos mayores puntos de encuentro entre nuestros resultados y los de estudios previos en las opiniones de la formación de grado y en la mirada de la utilidad de la investigación. Los estudiantes de Magisterio aprecian favorablemente la formación en investigación que han recibido, pero esperarían que su preparación en el tema fuera mucho más profunda, detallada y que les permitiera tener un contacto más precoz con la investigación. Coincidentemente con estos hallazgos, Demircioglu (2008) y MacDonald et al. (2001) sugieren que los programas de grado necesitan ser revisados con el objetivo de lograr una mayor relevancia de las asignaturas vinculadas con la investigación. Por su parte, los docentes del estudio de Jyrhämä et al. (2008) y de Demircioglu (2008) mejoran la imagen que tienen de la investigación una vez que realizan una asignatura de métodos y valoran positivamente lo que han podido conocer de investigación en el grado. Lo mismo sucede con los estudiantes de Magisterio de este estudio que ya cursaron la asignatura de métodos, quienes aprecian lo que han aprendido de investigación, aunque asumiendo que su formación en el tema podría ser mejor.

En los hallazgos de los estudios de Gitlin et al. (1999) o de MacDonald et al. (2001) se destaca que las investigaciones son útiles cuando proporcionan sugerencias concretas que refuercen y mejoren inmediatamente la práctica docente. En este punto encontramos coincidencias parciales con lo indicado por los estudiantes de Magisterio de esta investigación, quienes mencionan que la investigación debe darles la posibilidad de adquirir nuevos conocimientos factibles de trasladar a su aula. Si bien sus comentarios no son tan restrictivos en relación a la utilidad "inmediata" de los saberes al aula, sí son enfáticos al señalar que necesitan que la investigación sea capaz de relacionar sus contenidos con la práctica.

\section{CONCLUSIONES}

Quizá el hecho más llamativo, a la par de esperanzador, es la percepción razonablemente positiva que los estudiantes del grado de Maestro de Educación Primaria tienen de la investigación educativa y de sus posibilidades para mejorar la educación. Este hecho contrasta poderosamente con la imagen devastadoramente negativa que tienen los docentes en ejercicio sobre la investigación, tanto en España (p. ej., Murillo \& Perines, 2016; Murillo, Perines \& Lomba, 2016), como de otros países (p.ej., Anwaruddin, 2015; Cain, 2015; Drill 
et al., 2012; Hemsley-Brown Sharp, 2003; Ion \& Iucu, 2014; Witherow, 2011). Lo anterior nos lleva a dos planteamientos diferenciados.

De un lado es necesario seguir trabajando para conocer dónde y en qué momento se produce esta ruptura entre la docencia y la investigación. La hipótesis que aquí planteamos, y que deberá ser objeto de una investigación específica sobre el tema, es que en la incorporación a la escuela del docente se produce una asimilación de una cultura escolar de rechazo a la investigación. Así, es posible que el fenómeno en esos momentos pase de ser una "percepción" a un "imaginario". En otras palabras, frente la percepción entendida como una construcción individual generada desde la experiencia de diversos fenómenos, un imaginario es una construcción social a partir de significaciones que no corresponden a elementos racionales y que solo existen como una construcción de un ente colectivo impersonal y anónimo (Castodiaris, 1997).

El necesario entendimiento entre la investigación y la docencia pasa por un esfuerzo de desmontar prejuicios, más o menos razonables o justificados, que muestren las aportaciones de la investigación a la mejora de la práctica.

La segunda idea conclusiva de esta investigación es que desde la formación de profesorado se está consiguiendo ese acercamiento entre la investigación y la docencia. Cierto es que hay mucho que hacer (las percepciones críticas de los estudiantes hacia, por ejemplo, la materia de Métodos de Investigación son más que claras), pero parece que hay evidencias empíricas que afirman que esa distancia no se genera en la Universidad. Cierto es que no se consigue acabar con el problema, pero al menos no lo genera. En todo caso, hay que destacar la importancia de las materias que acercan la investigación al futuro docente.

Esta investigación se encuadra en una línea de trabajo que intenta conocer y comprender las relaciones entre docencia e investigación educativa. Relaciones difíciles, según todas las evidencias, pero por eso necesitadas de una mayor profundización. Este trabajo se ha focalizado en los docentes de educación primaria en formación, y de ahí viene el primer reto: habría que replicarlo para futuros docentes de Educación Infantil y de Educación Secundaria. Otra característica, en este caso limitación organizativa, es que solo se han estudiado alumnos de una universidad. Sería bueno hacerlo en otros contextos y universidades. La tercera característica, en este caso fortaleza, del estudio es el enfoque fenomenográfico utilizado, lo que nos ha permitido comprender en profundidad las percepciones de los participantes. En todo caso, un estudio de carácter ex post facto, con una mayor muestra, podría complementar esos resultados.

Si queremos que la investigación consiga impactar realmente en la mejora de la educación es necesario cambiar esa "terrible reputación" que nos decía Kaestle (1993). Cierto es que la investigación tiene mucho que mejorar: los temas a estudiar, las metodologías utilizadas, la forma de comunicar los resultados, las estrategias de implicar al profesorado, pero no lo es menos que tenemos que conocer las causas de esa brecha para acabar con ella. Es necesaria una mejor educación para una sociedad más justa y equitativa, y la investigación debe contribuir a ello.

\section{REFERENCIAS BIBLIOGRÁFICAS}

Anwaruddin, S.M. (2015). Teachers' engagement with educational research: Toward a conceptual framework for locally-based interpretive communities. Education Policy Analysis Archives, 
23(40), 1-25.

Ball, D.L., \& Forzani, F.M. (2009). The work of teaching and the challenge for teacher education. Journal of Teacher Education, 60(5), 497-511.

Berliner D.C. (2002). Educational research: the hardest science of all. Educational Researcher, 31(8), $18-20$.

Beycioglu, K., Ozer, N., \& Ugurlu, C.T. (2010). Teachers' views on educational research. Teaching and Teacher Education, 26(4), 1088-1093.

Bowden, J.A., \& Walsh, E. (2000). Phenomenography. Phenomenography, 1, 154-169.

Broekkamp, H., \& Van Hout-Wolters, B. (2007). The gap between educational research and practice: A literature review, symposium, and questionnaire. Educational Research and Evaluation, 13(3), 203-220.

Burkhardt, H., \& Schoenfeld, A.H. (2003). Improving educational research: Toward a more useful, more influential, and better-funded enterprise. Educational Researcher, 32(9), 3-14.

Cain, T. (2015). Teachers' engagement with published research: addressing the knowledge problem. The Curriculum Journal, 26(3), 488-509.

Castoriadis, C. (1997). El imaginario social instituyente. Zona Erógena, 35, 1-9.

Counsell, C., Evans, M., McIntyre, D., \& Raffan, J. (2000). The usefulness of educational research for trainee teachers' learning. Oxford Review of Education, 26(3-4), 467-482.

De la Orden, A., \& Mafokozi, J. (1999). La investigación educativa: naturaleza, funciones y ambiguiedad de sus relaciones con la práctica y la política educativas. Revista de Investigación Educativa, 17(1), 7-29.

Demircioglu, I.H. (2008). Learning How to Conduct Educational Research in Teacher Education: A Turkish Perspective. Australian Journal of Teacher Education, 33(1), 1-17.

Drill, K., Miller, S., \& Behrstock-Sherratt, E. (2012). Teachers' Perspectives on Educational Research. Naperville, IL: American Institutes for Research.

Edwards, T. (2000). 'All the Evidence Shows...': reasonable expectations of educational research. Oxford Review of Education, 26(3-4), 299-311.

Elliot, J. (2001). Making evidence-based practice educational. British Educational Research Journal, 27(5), 555-574.

Feuer, M.J., Towne, L., \& Shavelson, R.J. (2002). Scientific culture and educational research. Educational Researcher, 31(8), 4-14.

Gitlin, A., Barlow, L., Burbank, M.D., Kauchak, D., \& Stevens, T. (1999). Pre-service teachers' thinking on research: Implications for inquiry oriented teacher education. Teaching and Teacher Education, 15(7), 753-769.

Glass, G.V. (1998). “The Vision Thing”: Educational Research and AERA in the 21st Century. Part 4: The Future of Scholarly Communications. Educational Researcher, 27(8), 35-37.

Gore, J.M., \& Gitlin, A.D. (2004). [Re] Visioning the academic-teacher divide: Power and knowledge in the educational community. Teachers and Teaching, 10(1), 35-58.

Gray, C. (2013). Bridging the teacher/researcher divide: Master's-level work in initial teacher education. European Journal of Teacher Education, 36(1), 24-38.

Hammersley, M. (2002). Educational research, policymaking and practice. London: Sage Publications.

Hammersley, M. (2014). Translating research findings into educational policy and practice: the virtues and vices of a metaphor. Nouveaux c@ hiers de la recherche en éducation, 17(1), 54-74.

Hargreaves, D.H. (1996). Teaching as a research-based profession: possibilities and prospects. Conferencia presentada en el Annual Lecture of the Teacher Training Agency (TTA). Londres: Reino Unido.

Hemsley-Brown, J., \& Sharp, C. (2003). The use of research to improve professional practice: A systematic review of the literature. Oxford Review of Education, 29(4), 449-470.

Howe, K.R. (1998). The interpretive turn and the new debate in education. Educational Researcher, 
27(8), 13-20.

Ion, G., \& Iucu, R. (2014). Professionals' perceptions about the use of research in educational practice. European Journal of Higher Education, 4(4), 334-347.

Joram, E. (2007). Clashing epistemologies: Aspiring teachers', practicing teachers', and professors' beliefs about knowledge and research in education. Teaching and teacher education, 23(2), 123135.

Jyrhämä, R., Kynäslahti, H., Krokfors, L., Byman, R., Maaranen, K., Toom, A., \& Kansanen, P. (2008). The appreciation and realization of research-based teacher education: Finnish students' experiences of teacher education. European Journal of Teacher Education, 31(1), 1-16.

Kaestle, C. (1993). The awful reputation of education research. Educational Researcher, 22(1), 23-31.

Kennedy, M.M. (1997). The connection between research and practice. Educational Researcher, 26(7), 4-12.

Korthagen, F.A. (2007). The gap between research and practice revisited. Educational Research and Evaluation an International Journal on Theory and Practice, 13(3), 303-310.

Labaree, D.F. (1998). Educational researchers: Living with a lesser form of knowledge. Educational Researcher, 27(8), 4-12.

Levin, B. (2013). To know is not enough: research knowledge and its use. Review of Education, 1, 2-31.

Lysenko, L.V., Abrami, P.C., Bernard, R.M., Dagenais, C., \& Janosz, M. (2014). Educational Research in Educational Practice: Predictors of Use. Canadian Journal of Education, 37(2), 1-26.

MacDonald, M., Badger, R., \& White, G. (2001). Changing values: what use are theories of language learning and teaching? Teaching and Teacher Education, 17(8), 949-963.

Marton, F. (1986). Phenomenography: A research approach to investigating different understandings of reality. Journal of Thought, 21(3), 28-49.

Montgomery, C., \& Smith, L.C. (2015). Bridging the Gap between Researchers and Practitioners. Die Unterrichtspraxis/Teaching German, 48(1), 100-113.

Muñoz-Repiso, M. (2004). ¿Sirve para algo la investigación educativa? Organización y gestión educativa: Revista del Fórum Europeo de Administradores de la Educación, 12(1), 8-14.

Muñoz-Repiso, M. (2005). Mejorar las relaciones entre investigación y práctica educativas. Avances en Supervisión Educativa, 1. Recuperado de https://avances.adide.org/index.php/ase/article/ view/163

Murillo, F.J. (2006). Retos de la innovación para la investigación educativa. En T. Escudero y A. D. Correa (Coords.), Innovación e investigación educativa: algunos ámbitos relevantes (pp. 2354). Madrid: La Muralla.

Murillo, F.J. (2011). Hacer de la Educación un ámbito basado en evidencias científicas. REICE. Revista Iberoamericana sobre Calidad, Eficacia y Cambio en Educación, 9(3), 1-11.

Murillo, F.J., \& Perines H. (2016). Cómo los docentes no universitarios perciben la investigación educativa. Revista Complutense de Educación, en prensa.

Murillo, F.J., Perines, H., \& Lomba, L. (2016). La comunicación de la investigación educativa. Una aproximación a la percepción de los artículos académicos y de difusión por parte de docentes no universitarios. Profesorado. Revista de Curriculum y Formación de Profesorado, en prensa.

Pendry, A., \& Husbands, C. (2000). Research and practice in history teacher education. Cambridge Journal of Education, 30(3), 321-334.

Richardson, J.T. (1999). The concepts and methods of phenomenographic research. Review of Educational Research, 69(1), 53-82.

Rust, F. (2009). Teacher research and the problem of practice. The Teachers College Record, 111(8), $1882-1893$

Sancho, J.M. (2010). Del sentido de la investigación educativa y la dificultad de que se considere para guiar las políticas y las prácticas. REICE. Revista Iberoamericana sobre Calidad, Eficacia y Cambio en Educación, 8(2), 34-46. 
Slavin, R.E. (2002). Evidence-based education policies: transforming educational practice and research. Educational Researcher, 31(7), 15-21.

Sroufe, G.E. (1997). Improving the "awful reputation" of education research. Educational Researcher, 26(7), 26-28.

Thomas, G., \& Pring, R. (Eds.). (2004). Evidence-Based Practice in Education. Maidenhead: Open University Press.

Tom, A.R. (1997). Redesigning teacher education. Albany, NY: State University of New York Press.

Tooley, J., \& Darby, D. (1998). Educational Research: a critique. London: OFSTED.

Trinder, L., \& Reynolds, S. (Eds.). (2000) Evidence-Based Practice: a critical appraisal. Oxford: Blackwell Science.

van der Linden, W., Bakx, A., Ros, A., Beijaard, D., \& van den Bergh, L. (2015). The development of student teachers' research knowledge, beliefs and attitude. Journal of Education for Teaching, 41(1), 4-18.

van der Linden, W., Bakx, A., Ros, A., Beijaard, D., \& Vermeulen, M. (2012). Student teachers' development of a positive attitude towards research and research knowledge and skills. European Journal of Teacher Education, 35(4), 401-419.

Vanderlinde, R., \& van Braak, J. (2010). The gap between educational research and practice: views of teachers, school leaders, intermediaries and researchers. British Educational Research Journal, 36(2), 299-316.

Wagner, J. (1997). The unavoidable intervention of educational research: A framework for reconsidering researcher-practitioner cooperation. Educational Researcher, 26(7), 13-22.

Winch, C., Oancea, A., \& Orchard, J. (2015). The contribution of educational research to teachers' professional learning: philosophical understandings. Oxford Review of Education, 41(2), 202-216.

Witherow, K. (2011). Research use and the impact in secondary schools (Tesis doctoral). University of Toronto, Toronto.

Zeichner, K. (1995). Beyond the divide of teacher research and academic research. Teachers and Teaching: Theory and Practice, 1(2), 153-172.

Zeichner, K. (2010). Rethinking the connections between campus courses and field experiences in college-and university-based teacher education. Journal of Teacher Education, 61(1-2), 89-99.

Zeuli, J.S. (1994). How do teachers understand research when they read it? Teaching and Teacher Education, 10(1), 39-55. 